International comparisons of the quality of health care

\title{
International comparisons of the quality of health care: what do they tell us?
}

\section{K Walshe}

\section{What are international comparisons of healthcare quality for? Why and when should we want to compare the performance of health systems across countries and what should we do with the results?}

a

nternational comparisons of quality, access, and cost in health care are all the rage. The publication of the World Health Organization's World Health Report on health system performance in $2000,{ }^{1}$ in which the health systems of 191 countries were ranked using an aggregate measure based on several dimensions-population health, health inequalities, responsiveness, distribution of responsiveness, and financial fairness - stimulated worldwide attention to the business of measuring and comparing health system performance and resulted in a storm of controversy. The WHO methodology was fiercely attacked and equally stoutly defended. ${ }^{2-4}$ The report aroused anger, especially among commentators from countries which had done badly such as the US. The US outspends almost every other country on health care and prides itself on the sophistication and enterprise of its health system, but was humiliatingly ranked $37 \mathrm{th}$, bottom of all industrialised countries and below places such as Greece, Portugal, and Ireland. For healthcare providers in many countries like the UK who have been subjected to the publication of various hospital league tables, mortality comparisons and other performance measures over recent years, there was a certain "schadenfreude" to be had in watching the reactions of defensiveness, discomfort, and denial from national politicians and policymakers to the WHO report. It seems that nations respond to comparative performance data in much the same way as healthcare organisations. ${ }^{5}$

With rather less media attention, the Organisation for Economic Cooperation and Development (OECD)-which, for some time, has maintained an extensive database of comparative health system performance indicators - this year published an edited collection of papers on international comparisons of health system performance which comprehensively summarised the state of the $\operatorname{art}^{6}{ }^{6} \mathrm{It}$ gives a cautious and contingent account

of the conceptual and methodological challenges involved in developing and using performance measures across international boundaries, and provides an authoritative and highly readable insight into some of the solutions. But it still leaves largely unanswered the question of what international comparisons are for? Why and when should we want to compare the performance of health systems across countries and what should we do with the results?

\section{"we have to keep reminding those who produce international comparative datasets and indicators that their value . . . must be the contribution they make to improvement"}

The work of Marshall and colleagues in this issue of $Q S H C^{7}$ provides a neat case study illustrating this problem of the purpose of international comparisons. They show that taking indicators developed in one country and simply using them in another is probably inappropriate and unwise, given the differences in clinical practice and context which exist. In their study only about $56 \%$ of the quality indicators developed for 18 common primary care conditions in the USA made it into the indicators adapted for the UK. Furthermore, they found that differences in data collection systems and healthcare financing and organisation meant that straightforward comparisons were difficult. They conclude that transferring indicators is possible but needs to be done carefully and to take account of differences in context. But, valuable though these points are, they are all simply questions of methodology. The big issue-why should I want to take indicators developed in the US and use them elsewhere, and what would I learn from doing so which might improve the quality of care-is not explicitly addressed.

I would contend that the business of performance measurement is too often led by the technical and statistical wizards who develop the systems of measurement, and not by the ordinary people who need to use those systems of measurement to do their job in managing healthcare organisations. As a result, we get a lot of measurement but not much understanding, lots of data but little change. The measurement process is driven by the information and the clever measures we can build with it, not by our ideas about what needs improving in our healthcare system, and how we might do it. ${ }^{8}$

More radically, I would suggest that we don't need international comparisons. Rather, what we need is international learning, by which I mean the capacity and capability for healthcare policymakers and others to learn from experience elsewhere-good and badusing the health systems of other countries as the testing grounds for innovations before they are piloted or adopted at home. 'If that process needs comparative data, then all well and good, but the data and indicators should be an explicit product of the need for learning, targeted on the issue in hand. When international comparative data are assembled and presented on the off chance that they might reveal something interesting, it quickly degenerates into a fishing expedition for differences (and, if you look long and hard enough, you will always find some) or an exercise in point scoring and trumping.

The British NHS is more willing now than ever before to look abroad for ideas and lessons on improvement-an encouraging trend which hopefully might also be reflected in the future in other countries. ${ }^{10}$ For example, in planning to move towards a new system of "payment by results" for healthcare providers, the Department of Health in England has drawn heavily on the experience of other European countries such as Austria, Denmark and Norway in implementing case mix based reimbursement mechanisms and tariffs. ${ }^{11}$ With the aim of improving disease management in primary care, the Department of Health and its Modernisation Agency are bringing in expertise in IT and care pathways from a number of leading US health maintenance organisations. ${ }^{12}$ The British improvement collaboratives programme has drawn heavily for inspiration and expertise on the US experience of setting up and running collaboratives. ${ }^{13}$ In each of these endeavours, comparative data can and do play a supporting role, but are a tool rather than the purpose of the process.

If countries are serious about collaborating and learning from each others' healthcare systems, then international comparisons of the quality of health care can be enormously valuable in directing and focusing that learninghighlighting and spreading good practice. But, if comparative data are used 
mainly to rank countries like teams in a football league, ${ }^{14}$ and to fuel a dialogue of the deaf about whose system is better than whose, it will be a profoundly unhelpful and unproductive use of resources which could be spent in so many better ways. Ultimately, we have to keep reminding those who produce international comparative datasets and indicators that their value and the sole metric of their worth must be the contribution they make to improvement.

Qual Saf Health Care 2003;12:4-5

\section{Author's affiliation}

K Walshe, Director of Research and Reader in Public Management, Manchester Centre for Healthcare Management, University of Manchester, Manchester M13 9PL, UK;

kieran.walshe@man.ac.uk

\section{REFERENCES}

1 World Health Organization. The World Health Report 2000: health systems, improving performance. Geneva: WHO, 2000.

2 Navarro V. Assessment of the World Health Report 2000. Lancet 2000;356: 1598-601.

3 Murray C, Frenk J. World Health Report 2000: a step towards evidence based policy. Lancet 2001;357: 1698-700.

4 Blendon RJ, Kim M, Benson JM. The public versus the World Health Organization on health system performance. Health Affairs 2001;20:10-20.

5 Marshall MN, Shekelle PG, Leatherman S, et al. The public release of performance data: what do we expect to gain? A review of the evidence. JAMA 2000;283:1866-74.

6 Smith P, ed. Measuring up: improving health system performance in OECD countries. Paris: OECD, 2002

7 Marshall MN, Shekelle PG, McGlynn EA, et al. Can health care quality indicators be transferred between countries? Qual Saf Health Care 2003;12:8-12.
8 Berwick DM. A primer on leading the improvement of systems. BM 1996:312:619-22

9 Walshe K. Don't try this at home: health policy lessons for the NHS from the United States. Econ Affairs 2001;21:31-5.

10 Brown LD. Exceptionalism as the rule? US health policy innovation and cross-national learning. J Health Politics Policy Law 1998:23:35-51.

11 Department of Health. Reforming NHS financial flows: introducing payment by results. London: Department of Health, 2002.

12 Anonymous. US groups to work with PCTs on pilot project. Health Serv J 2002; 1 12:5.

13 Bate SP, Robert G, Mcleod H. Report on the breakthrough collaborative approach to quality and service improvement within four regions of the NHS. Birmingham: Health Services Management Centre, University of Birmingham, 2002

14 Appleby J, Street A. Health system goals: life, death and . . . football. J Health Serv Res Policy $2001 ; 6: 220-5$.

\section{Safe treatment or a shorter wait: Hobson's choice?}

\section{Angela Coulter}

\section{The British government hopes that the Patient Choice scheme recently introduced by the NHS will lead to improvements in waiting times and quality.}

W hen Henry Ford offered customers of his Model $\mathrm{T}$ car a choice of "any colour so long as it is black" there was no need for sophisticated techniques to check out their preferences. Until very recently options for patients in the British NHS were just as restricted as Ford's colour range. Hobson's choice was what was on offer-that is, no choice at all, you get what you're given. The situation is about to change dramatically following the government's recent launch of the Patient Choice scheme. ${ }^{1}$ Patients who have been on the waiting list for elective surgery for a specified period (initially 6 months) will be offered a choice of staying on the list until an operation slot becomes available at their "home" hospital or being treated faster elsewhere-in another NHS hospital, a private hospital, or even abroad. Those people considering such a choice will need to trade off the certainty of an earlier operation date, possibly far away from family and friends, against the perceived advantages of continuity of care in a familiar environment.

In this issue of QSHC Ross and colleagues report on a study in which they asked participants to make a trade off between prompt access and complication risk. ${ }^{2}$ In this "stated preference" game participants were not offered the chance to travel elsewhere to find what many might have preferred-namely, fast access to safe treatment. Instead, the scenario assumed they would stay at their local hospital and be given a strictly limited choice between a long wait for cataract surgery performed by a consultant or a shorter wait for treatment by a trainee with a higher complication rate.

The notion that the way to speed up access is to offer less safe care would be profoundly disturbing to most patients if they knew this was being mooted. The fact that a high proportion of those invited to participate in this study were willing to play the game and make the trade offs, albeit in a hypothetical situation, is indicative of the level of public concern in the UK about long waiting times for elective surgery. Forced to contemplate spending 16 months with poor vision, some people viewed a $10 \%$ risk of harm from cataract surgery as a lesser evil.
The doctor's grade and experience did not have an important independent effect in this study, presumably because participants were unaware of the association between grade and complication rates. Indeed, the authors tell us that it is standard practice to withhold information on surgeon grade, so patients are deliberately kept in the dark.

For ethical and practical reasons this situation should not be allowed to continue. Patients have a right to know about the training and past performance of those to whom they are about to entrust themselves. Those responsible for supervising trainee surgeons should be required to do everything in their power to ensure that patients are not harmed by the trainees' relative lack of skill. Perhaps publication of performance measures, including comparisons by surgeon grade, would provide a powerful incentive to raise quality standards. ${ }^{3}$

Most British patients currently have no information about the success rates of their surgeons, and no way of finding out because the data are not yet published. Even finding accurate information about waiting times is a difficult task for most people. Many people on surgical waiting lists have little idea of when they are likely to receive their operation. If the Patient Choice initiative is to succeed in its aim of reducing waiting times by empowering patients, these information problems will have to be solved. But will patients want to use the information to make informed choices?

Information on process and outcome indicators in different hospitals has been a feature of the American scene for some years, but as yet there is little evidence that it is having an impact on patient behaviour. Despite the fact that the indicators reveal considerable variations in 
performance between hospitals, few patients are aware of the data and even fewer seem to use them to choose providers. ${ }^{4}$ A number of explanations have been proffered for this lack of impact. Patients may be unaware of variations in quality so they don't seek information about the "best" providers. Some don't believe choice is possible or don't want to exercise it. ${ }^{6}$ And often the information is not available at the time it is needed, or is not provided in a sufficiently user friendly form. ${ }^{7}$ It remains to be seen whether the British public will respond with the same degree of indifference to the publication of performance information. A key issue will be whether greater transparency enhances or undermines public confidence in the NHS. For example, will better information and increased choice make it harder to persuade people to be treated by trainees?

The British government hopes that patient choice will be the grit in the oyster that leads to improvements in waiting times and quality. The roll-out of this experiment will tell us whether it is realistic to hope for such pearls or whether Hobson's choice is our inevitable fate.

Qual Saf Health Care 2003;12:5-6

\section{Author's affiliation}

A Coulter, Picker Institute Europe, King's Mead House, Oxpens Road, Oxford OXI 1RX, UK: angela.coulter@pickereurope.ac.uk

\section{REFERENCES}

1 Department of Health. Extending choice for patients. London: Department of Health, 2001.

2 Ross M-A, Avery AJ, Foss AJE. Views of older people on cataract surgery options: an assessment of preferences by conjoin analysis. Qual Saf Health Care 2003;12:13-17

3 Berwick DM. Public performance reports and the will for change. JAMA 2002;288:15234.

4 Marshall MN, Shekelle PG, Leatherman S, et al. The public release of performance data: al. The public release of performance data: what do we expect to gain? A review of the JAMA 2000:283:1866-74.

5 Schneider EC, Lieberman T. Publicly disclosed information about the quality of health care: response of the US public. Qual Health Care 2001;10:96-103.

6 Hoy EW, Wicks EK, Forland RA. A guide to facilitating consumer choice. Health Affairs 1996:15:9-30.

7 Hibbard JH, Slovic P, Peters E, et al. Is the informed-choice policy approach appropriate for Medicare beneficiaries? Health Affairs 2001;20: 199-203.

\section{Influence of attitudes and behaviour of GPs on prescribing costs}

\section{J Szecsenyi}

\section{The relationship between the attitudes of health professionals and their behaviour is complex. How can it be changed?}

R ising prescribing costs are a major concern in modern healthcare systems worldwide. Wherever costs are analysed and combined with any kind of quality measures, large variations between practices can be seen which cannot be explained fully by the underlying morbidity of the patients treated in these practices. For this reason, the knowledge, attitudes, and beliefs of doctors themselves has become the focus of research.

Since the early work of Parish ${ }^{1}$ we have known that prescribing has an irrational as well as a rational basis. Balint et al showed that writing a prescription is an easy way of cutting the consultation time for overworked doctors. Howie ${ }^{3}$ in his study of clinical judgement and antibiotic use in general practice demonstrated the influence of situational factors on prescribing decisions. The personal views of the doctor can also play a similar role ${ }^{4}$; often GPs think that patients expect to get a prescription at the end of the consultation. In European countries such as Germany, Belgium, and Switzerland which have no fixed list system, this may lead to a fear of losing patients, although large patient surveys in these countries have shown that more patients change their GP because of overprescribing than because they think their doctor tries to keep down prescribing costs. ${ }^{5}$

The paper by Watkins et $a l^{6}$ in this issue of QSHC identifies more factors and summarises some well known findings on the basis of a cross sectional study. The influence of the pharmaceutical industry again becomes apparent. We know that it is pervasive but almost invisible to individuals-but what else could we expect? Why should an industry invest billions of dollars per year in changing behaviour their way if they did not see this is as an effective intervention?

A question which often arises in this context is whether we need "sticks or carrots" to change prescribing patterns. The answer is unclear. What we now know from research on implementing change is that a multimodal approach, tailored to the individual needs of the doctor, is likely to be more effective than a single "one shot" intervention. ${ }^{8}$ We have to remember that not all doctors are the same, and that they are part of a complex health system, on the one hand, and part of a complex interaction with the patient on the other. Prescribing decisions, like all medical decisions, are the result of an interaction between the GP and patient, so their "rational" basis will always be affected by a combination of attitudes, beliefs, and knowledge of the two parties involved. This is why we have to understand the personal needs, beliefs, and attitudes of GPs and patients in order to give them the kind of support which might be most effective for them. In future this could mean that interventions on the doctor's side will have to be tailored, not only on a regional basis, but also to the individual practice and the individual doctor. An intervention would mean a flexible framework which gives information and feedback in an environment with trust and support combined with financial and other incentives. "Sticks" will then be necessary only for those few (if any) who are unwilling to do anything. As Bradley ${ }^{9}$ has recently pointed out, we need greater insight from qualitative research and must adopt the findings such as those of Watkins et al to improve our interventions. I am sure we can.

Qual Saf Health Care 2003;12:6-7

Author's affiliation

J Szecsenyi, Department of General Practice and Health Services Research, Medical Hospital and Polyclinic, University of Heidelberg,

D-69120 Heidelberg, Germany;

Joachim_Szecsenyi@med.uni-heidelberg.de

\section{REFERENCES}

1 Parish PA. Sociology of prescribing. Br Med Bull 1974;30:214-7.

2 Balint M, Hunt J, Joyce D, et al. Treatment or diagnosis. A study of repeated prescriptions in general practice. London: Tavistock Publications, 1970

3 Howie JG. Clinical judgement and antibiotic use in general practice. BM 1976;1061-4.

4 De Sutter A, de Meyere J, de Maesener J, et al. Antibiotic prescribing in acute infections of the nose or sinuses: a matter of personal habit? Fam Pract 2001;18:209-13.

5 Klingenberg A, Szecsenyi J. Not every visit needs a prescription. Patients' expectations towards phamacotherapy in general practice (in German). Goettingen: AQUA, 1998. 
6 Watkins C, Harvey I, Carthy P, et al. Attitudes and behaviour of general practitioners and their prescribing costs: a national cross sectional survey. Qual Saf Health Care 2003;12:29-34.
7 Wazana A. Physician and the pharmaceutical industry. Is a gift ever just a gift? JAMA 2000;283:373-80.

$8 \mathrm{Grol}$ R. Successes and failures in the implementation of evidence-based guidelines for clinical practice Med Care 2001;39(Suppl 2):1146-54.

9 Bradley CP. Insights from qualitative research are needed to improve GP prescribing. Eur J Gen Pract 2002;8:3-5.

Bimonthly publication

\section{QSHC 2003: now bimonthly}

\section{F Moss}

\section{Another step forward!}

$$
\mathrm{T}_{\mathrm{o}}^{\mathrm{h}}
$$

his issue marks the first anniversary of the journal's transition from $Q H C$ to QSHC and the first issue of a new bimonthly publication schedule. We now have more readers and more people than ever are visiting the web site. The most popular papers-as judged by "hits on the web" - have been "A preliminary taxonomy of medical errors in family practice" by Dovey et al, ${ }^{1}$ followed by "The need for risk management to evolve to assure a culture of safety" by Kuhn and Youngberg. ${ }^{2}$ But, as well as attracting more readers, more authors have submitted papers for consideration for publication, and more papers have been accepted. So, to enable us to keep up, the journal is now bimonthly. There will be fewer pages in each issue-down from 96 to 80 -but with six issues a year we will publish more pages and more papers!

We are now fully electronic: all papers are submitted and reviewed electronically. There have been a few teething problems and our apologies to those who have found the system difficult to use. But these glitches are being resolved so that the system really can meet the needs of all users - authors and reviewers, as well as editors. Anyone who wants to submit a paper should visit the author area on the web site.
We have hardly begun to use the enormous potential of web based publishing and online access. More people have posted electronic responses to articles published. But, while an increase of $200 \%$ might be construed as a success, we have only just hit double figures! We welcome your feedback and comments and suggestions.

Qual Saf Health Care 2003;12:7

\section{Author's affiliation}

F Moss, Editor-in-Chief

\section{REFERENCES}

1 Dovey SM, Meyers DS, Phillips RL Jr, et al. A preliminary taxonomy of medical errors in family practice. Qual Saf Health Care 2002; 11:233-8

2 Kuhn AM, Youngberg BJ. The need for risk management to evolve to assure a culture of safety. Qual Saf Health Care 2002;11:158-62. 